\title{
LONGEVIDADE E REPRODUÇÃO DE Cryptolestes pusillus (SCHOENHERR) (COL., CUCUJIDAE) APÓS IRRADIAÇÃO GAMA
}

\author{
F.M. WIENDL; V. ARTHUR \\ Centro de Energia Nuclear na Agricultura- CENA/USP - C.P. 96 - CEP:13400-970 - Piracicaba,SP \\ J.A. WIENDL \\ Informatt - Informatica Mattiolli - Piracicaba, SP \\ F.G. PELUTZEN \\ Katosila Uni. Patamanperäntie, Kukonkylä, Uusimaa, Finland
}

\begin{abstract}
RESUMO: Insetos adultos da especie Cryptolestes pusillus (Schoenherr) foram irradiados com idade entre 2 e 7 dias, com as seguintes doses de radiaçóes gama do Cobalto-60: 0 (test.); 12,5; 25,0; 50,0; 100,0; 200,0; 500,0; 1000,0; 2500,0 e $5000,0 \mathrm{~Gy}$, sob uma taxa de dose de 13,2 Gy por minuto. Após a irradiação os insetos foram mantidos em dois grupos, um à temperatura de $25^{\circ} \mathrm{C}$ e o outro a $30^{\circ} \mathrm{C}$, sob umidade relativa entre 60 e $80 \%$. Observou-se a cada dois dias a mortalidade destes insetos, concluindo-se que as vidas médias foram respectivamente as seguintes na temperatura de $25^{\circ} \mathrm{C}: 65,1 ; 67,7 ; 78,8 ; 81,5 ; 20,7 ; 12,3 ; 12,5 ; 9,2 ; 5,8$ e 2,6 dias. Na temperatura de $30^{\circ} \mathrm{C}$ as vidas médias foram: 67,$5 ; 119,5 ; 160,3 ; 66,2 ; 10,2 ; 9,2 ; 9,4 ; 6,6 ; 6,3$ e 2,2 dias. Igualmente a cada dois dias foram observados os nascimentos da primeira geração filial, chegando-se aos seguintes resultados quando a temperatura foi de $25^{\circ} \mathrm{C}: 19,11,14,16,3,0,0,0,0$ e 0 individuos. A $30^{\circ} \mathrm{C}$ foram os seguintes os números dos indivíduos nascidos: $299,227,118,31,7,3,5,0,0$ e 0 , conforme as doses de radiaçāo gama. Concluiu-se que conforme a temperatura a radiohormesis se manisfesta $\mathrm{em}$ menor grau $\left(25^{\circ} \mathrm{C}\right)$ ou grau mais elevado $\left(30^{\circ} \mathrm{C}\right)$ quando as observaçōes se referem à longevidade. Não se observaram efeitos positivos de radiohormesis quanto a reprodução no presente experimento.
\end{abstract}

Descritores: longevidade, reprodução, radiações gama, radiohormesis, desinfestação, Cryptolestes pusillus.

\section{LONGEVITY AND REPRODUCTION OF Cryptolestes pusillus (SCHOENHERR) (COL., CUCUJIDAE) AFTER GAMMA IRRADIATION}

\begin{abstract}
Adults of the species Cryptolestes pusillus (Schoenherr) were irradiated at ages between 2 and 7 days after emergency, with doses of: 0 (control), 12.5, 25.0, 50.0, 100.0, 200.0, 500.0, 1000.0, 2500.0 and 5000.0 Gy of gamma radiations of Cobalt-60, at a dose rate of $13.2 \mathrm{~Gy}$ per minute. After irradiation the insects were divided into two groups, one maintained at $25^{\circ} \mathrm{C}$ and the other at $30^{\circ} \mathrm{C}$, at a relative humidity between 60 and 80 percent. Every two days the insects were observed for mortality. The medium life spans were respectively, at the temperature of $25^{\circ} \mathrm{C}$ : 65.1, 67.7, 78.8, 81.5, 20.7, 12.3, 12.5, 9.2, 5.8 and 2.6 days. At the temperature of $30^{\circ} \mathrm{C}$ the medium life spans were 67.5, 119.5, 160.3, 66.2, 10.2, 9.2, 9.4, 6.6, 6.3 and 2.2 days. Similar to the mortality observations, the reproduction was also determinated, and the number of adults emerged was respectively to the radiation doses at $25^{\circ} \mathrm{C}: 19,11,14,16,3,0,0,0,0$ and 0 individuals. At $30^{\circ} \mathrm{C}$ the number of emerged adults was 299, 227, 118, 31, $7,3,5,0,0$ and 0 respectively. It was concluded that radiohormesis is observed to a lower extend depending on the temperature, more at higher temperatures and less at lower temperatures, if we consider longevity. No positive effect of radiohormesis could be observed in relation to reproduction in the present study.

Key Words: longevity, reproduction, gamma radiations, radiohormesis, disinfestation, Cryptolestes pusillus

\section{INTRODUÇÃO}

Uma das pragas que atacam farelos, farinhas ou mesmo grãos ofendidos por pragas primárias é Cryptolestes pusillus (Schoenherr). Embora sem causar prejuízos acentuados em grãos

inteiros, é de efeito devastador em farinhas onde conspurca e torna imprestável o produto. Seu ciclo evolutivo se dá em um pouco mais de três semanas sob condições favoráveis, com alto poder reprodutivo, sendo que os adultos podem viver de 6 a 8 meses.
\end{abstract}


Com a atual tecnologia é dificílimo seu controle pelos métodos usuais de fumigação, pois a penetração de gases tóxicos em farelos e farinhas se dá com muita dificuldade. Desta forma é mesmo impossível o controle se o produto já estiver empacotado, em embalagens plásticas ou de papel para o consumidor final. Somente o controle, preferencialmente em suas fases iniciais por radiações ionizantes, é o único método viável e eficiente, sem prejuizos para o produto. Neste caso nada se introduz a não ser energia radiante que se dissipa imediatamente após aplicação, sem causar efeitos secundários.

Diversos autores entre eles o primeiro que trabalhou na aplicação de radiações ionizantes, HUNTER (1912) tentaram chegar a bons resultados com raios $X$, controlando as pragas de grãos armazenados. Embora este autor não tivesse êxito ao irradiar Sitophilus oryzae (L.), outro pesquisador RUNNER (1916), trabalhando com uma nova ampôla de raios $X$ conseguiu controlar Lasioderma serricorne ( $F$.) que atacava cigarros.

No Brasil, somente em 1960 GALLO publicou um trabalho citando a possibilidade de controlar Diatraea saccharalis e Ceratitis capitata, no intuito de esterilizar estas duas espécies e depois liberá-las nas culturas, aplicando a técnica do macho estéril.

Em 1968, WIENDL iniciou uma série de publicações sobre os efeitos das radiações gama do Cobalto-60 em insetos de grãos armazenados. Assim, em 1969, publicou tese compilando resultados obtidos na irradiação gama de Zabrotes subfasciatus. No ano de 1972 publicou os resultados obtidos com a irradiação de Sitophilus zeamais.

Desta forma vem-se observando os efeitos das radiações gama nas principais pragas de grãos armazenados, primárias e secundárias, também em nosso país.

Porém, em trabalhos de radiobiologia, tanto com vegetais ou animais podem muitas vezes ser observados efeitos à primeira vista contrários aos esperados pelos pesquisadores teóricos, pois ainda hoje é pensamento que a ionização por radiações em organismos vivos é sempre deletérica.

Mas a prática não confirma isto e sim, sob certas condições de baixas doses e taxas de irradiação aplicados nos organismos tratados possuem vezes sem conta uma longevidade muito maior, aliada muitas vezes a uma taxa de reprodução significativamente mais elevada. E plantas cultivadas produzem mais.
No Brasil o primeiro trabalho neste sentido foi o de VILHENA' realizado nos anos de 1976 a 1978, com radiohormesis em arroz, colhendo-se entre 58 e $65 \%$ mais em relação às sementes não irradiadas.

Publicado em 1987 um dos primeiros relatos acadêmicos em radiohormesis em nosso país também foi realizado com plantas e desta vez KIKUCHI (1987) observou que sementes de arroz tiveram sua germinação acelerada com a dose de 3,5 Gy.

No Reino Animal, pouco se tem publicado no nosso país quando se aborda o assunto relativo à hormesis. Embora haja muitos trabalhos que expõe dados de sua existência, quase nenhum autor considerou o fenomeno como sendo de importância. Talvez este fato não tenha chamado atenção aos autores por estes estarem preocupados em determinar as doses esterilizantes e letais de insetos pragas da agricultura, a fim de efetivar um controle não poluente, eficiente e econômico.

Assim, em 1969 WIENDL se refere ao assunto sem analisá-to. Obteve em Zabrotes subfasciatus uma maior longevidade dos carunchos adultos ao irradiá-los com as doses de 25 e $50 \mathrm{Krad}$ (250 e $500 \mathrm{~Gy}$ ). Esta longevidade chegou a ser $15 \%$ maior na primeira dose e $7 \%$ maior na dose de $500 \mathrm{~Gy}$.

O mesmo autor obteve resultados significativos que relata em trabalhos publicados no ano de 1972 obtendo uma eclosão significativamente maior, de 14 a $24 \%$ nas larvas de $S$. zeamays, quando seus ovos foram irradiados com doses de 3 e 6 Gy. Também a emergência de adultos foi de 10 a $20 \%$ maior quando se irradiaram larvas com doses de 3 a 9 Gy. E o fenômeno se repetiu quando foram irradiadas pupas, observando-se que o número de adultos emergidos é $11 \%$ maior quando a dose foi de $16 \mathrm{~Gy}$.

No exterior, foi LUCKEY (1980 e 1991) que se preocupou sobremaneira em coletar informações relacionadas com os efeitos de baixas doses de radiações ionizantes sobre seres vivos. Assim em $1980 \mathrm{fez}$ uma resenha onde expôs os dados de mais de 1200 trabalhos em radiohormesis. Neste primeiro volume sua maior preocupação foi a de relatar trabalhos em animais e plantas, de maneira mais generalizada sob aspectos da radiobiologia.

\footnotetext{
'VILHENA, M.T.M.B. Departamento de Energia NuclearEE/UFRS: Comunicação pessoal, 1988.
} 
No segundo tomo, publicado em 1991, seus estudos são mais abrangentes na área de radiobiologia de vertebrados superiores e o homem chegando a relatar de forma concisa os resultados de mais de 1000 publicações.

Um ponto que chama atenção é que o autor julga permissível uma razoável extrapolação de resultados obtidos em experimentos com animais inferiores como os insetos, para o homem. Aliás, convém lembrar, que inclusive aqueles autores que não são favoráveis a aceitar as conclusões dos que aceitam a radiohormesis, também se baseiam principalmente em animais quando descrevem efeitos deletéricos, extrapolando-os ao homem.

Assim surge uma completa revisão dos conceitos erroneamente arraigados a favor de outros que mostram claramente os efeitos benéficos das radiações, desde que sejam em baixas doses e baixas taxas.

\section{MATERIAIS E MÉTODOS}

Irradiaram-se insetos adultos da espécie Cryptolestes pusillus (Schoenherr) (Coleoptera, Cucujidae) com idade de 2 a 7 dias, após a emergência, não sexados, com as seguintes doses de radiações: 0 (testemunha); 12,$5 ; 25 ; 50 ; 100$; 200; 500; $1000 ; 2500$ e $5000 \mathrm{~Gy}$.

A fonte de radiações utilizada foi uma fonte de Cobalto-60, e as doses foram administradas sob uma taxa de 13,2 Gy por minuto.

Para cada dose foram feitas 10 repetições, contendo 5 insetos e aproximadamente três gramas de fubá de milho amarelo. As repetições foram agrupadas em duas séries, cada qual mantida em uma câmara, a $30^{\circ} \mathrm{C}$ e a outra a $25^{\circ} \mathrm{C}$, sob umidade relativa entre 60 e $80 \%$. Após irradiação foram contados e retirados os insetos mortos, operação esta repetida a cada dois dias, até total extinção de todos os insetos que compunham aquela determinada repetição. Para que houvesse possibilidade de se conhecer a quantidade da reprodução, a cada vinte dias os insetos eram tranferidos para um outro vidrinho, contendo também três gramas de alimento. Os vidrinhos irradiados da geração paterna (P) agora sem os insetos ficaram sob observação também a cada dois dias, contando-se e retirando-se os adultos nascidos da primeira geraçâo filial $\left(F_{1}\right)$.

\section{RESULTADOS E DISCUSSÃO}

As contagens foram realizadas entre 07/03/75 (data da irradiação) até 18/02/76, quando se registrou a morte do último inseto após 348 dias.

Os insetos adultos irradiados com doses crescentes de radiações gama e mantidos após a irradiação à temperatura de $25^{\circ} \mathrm{C}$, quando expostos a doses de $100 \mathrm{~Gy}$ ou mais elevadas, sofreram sensível diminuição em suas vidas médias, tornando-se esta tanto menor quanto maior a dose. Doses de até $50 \mathrm{~Gy}$ provocaram um aumento da vida média, chegando a mais de $25 \%$ quando irradiados com 50 Gy (TABELA 1).

TABELA 1. Total, média e porcentagem em relação às testemunhas de dias vividos por populações de adultos de $C$. pusillus irradiados com diferentes doses de radiações gama do Cobalto- 60 e mantidos à temperatura de 25 e $30^{\circ} \mathrm{C}$ após tratamento.

\begin{tabular}{lcccccc}
\hline DOSE & \multicolumn{3}{c}{$25^{\circ} \mathrm{C}$} & \multicolumn{3}{c}{$30^{\circ} \mathrm{C}$} \\
(Gy) & T & m & $\%$ & T & \multicolumn{1}{c}{ m } & $\%$ \\
\hline 0 & 1562 & 65,1 & 100,0 & 1688 & 67,5 & 100,0 \\
12,5 & 1628 & 67,7 & 104,0 & 2988 & 119,5 & 177,0 \\
25 & 1970 & 78,8 & 121,0 & 4008 & 160,3 & 237,5 \\
50 & 2038 & 81,5 & 125,2 & 1656 & 66,2 & 98,1 \\
100 & 518 & 20,7 & 31,8 & 256 & 10,2 & 15,1 \\
200 & 308 & 12,3 & 18,9 & 230 & 9,2 & 13,6 \\
500 & 312 & 12,5 & 19,2 & 234 & 9,4 & 13,9 \\
1000 & 230 & 9,2 & 14,1 & 164 & 6,6 & 9,8 \\
2500 & 146 & 5,8 & 8,9 & 158 & 6,3 & 9,3 \\
5000 & 64 & 2,6 & 4,0 & 56 & 2,2 & 3,3 \\
\hline
\end{tabular}

$\mathrm{T}=$ total

$\mathrm{m}=$ média

$\%=$ porcentagem em relação à dose de "0" Gy.

Fato semelhante ocorreu com os insetos mantidos à $30^{\circ} \mathrm{C}$ após irradiação, porém com a dose de $50 \mathrm{~Gy}$ a longevidade dos insetos era semelhante à da testemunha, diminuindo sensivelmente com a dose de $100 \mathrm{~Gy}$, fenômeno cada vez mais pronunciado com o aumento da dose. 
Porém, as doses de 12,5 e 25 Gy proporcionaram marcante aumento da vida dos insetos, chegando-se a 177 e $237,5 \%$ em relação à testemunha.

Em termos de reprodução, observou-se que as doses de radiações provocaram uma diminuição já a partir da menor dose empregada, conforme pode ser observado pelos resultados constantes na TABELA 2.

TABELA 2. Número total de adultos e porcentagem em relação às testemunhas da população filial proveniente de 25 adultos de $C$. pusillus irradiados com diferentes doses de radiações gama do Cobalto-60 e mantidos a $25^{\circ} \mathrm{C}$ e $30^{\circ} \mathrm{C}$ após irradiação.

\begin{tabular}{|c|c|c|c|c|}
\hline \multirow{2}{*}{$\begin{array}{l}\text { DOSE } \\
\text { (Gy) }\end{array}$} & \multicolumn{2}{|c|}{$25^{\circ} \mathrm{C}$} & \multicolumn{2}{|c|}{$30^{\circ} \mathrm{C}$} \\
\hline & Total & $\%$ & Total & $\%$ \\
\hline 0 & 19 & 100,0 & 299 & 100,0 \\
\hline 12,5 & 11 & 57,9 & 227 & 75,9 \\
\hline 25 & 14 & 73,7 & 118 & 39,5 \\
\hline 50 & 16 & 84,2 & 31 & 10,4 \\
\hline 100 & 3 & 15,8 & 7 & 2,3 \\
\hline 200 & 0 & 0,0 & 3 & 1,0 \\
\hline 500 & 0 & 0,0 & 5 & 1,7 \\
\hline 1000 & 0 & 0,0 & 0 & 0,0 \\
\hline 2500 & 0 & 0,0 & 0 & 0,0 \\
\hline 5000 & 0 & 0,0 & 0 & 0,0 \\
\hline
\end{tabular}

\section{CONCLUSÃO}

As radiações gama e temperatura de manutenção após a irradiação, influem sobremaneira na longevidade dos insetos adultos de C. pusillus, assim como em sua reprodução, mesmo na temperatura preferencial de $30^{\circ} \mathrm{C}$, desta espécie. Uma dose cinco vezes menor já é suficiente para causar o mesmo efeito quando os insetos são mantidos sob uma temperatura cinco graus menor, de $25^{\circ} \mathrm{C}$.

$\mathrm{O}$ efeito na longevidade é observado principalmente nos insetos submetidos a doses de até $50 \mathrm{~Gy}$, sendo que a temperatura de $30^{\circ} \mathrm{C}$ tem efeito pronunciado, pois observou-se que nesta temperatura a longevidade chegou a ser $237,5 \%$ quando os insetos foram irradiados com a dose de $25 \mathrm{~Gy}$, chegando a $121,0 \%$ na mesma dose quando após a irradiação, os insetos foram mantidos a $25^{\circ} \mathrm{C}$, se comparados com as testemunhas correspondentes.

\section{BIBLIOGRAFIA}

GALLO, D. Radioisótopos no controle das pragas. $\mathbf{O}$ Solo, Piracicaba, v.52, n.1, p.30-31, 1960.

HUNTER, W.D. Results of experiments to determine the effect of Roentgen rays upon insects.Journal of Economic Entomology, College Park, v.5, n.1, p.188, 1912.

KIKUCHI, O.K. Efeitos de baixas doses de radiação gama de Cobalto-60 sobre sementes de feijão, arroz e rabanete. São Paulo, 1987. 65p. Dissertação (Mestrado) - Instituto de Pesquisas Energéticas, Universidade de São Paulo.

LUCKEY, T.D. Hormesis with ionizing radiation, Boca Raton, CRC Press, 1980. 232p.

LUCKEY, T.D. Radiation hormesis. Boca Raton, CRC Press, 1991. 320p.

RUNNER, G.A. Effect of roentgen rays on the tobacco or cigarette-beetle and the results of new form of Roentgen Tube. Journal of Agricultural Research, v.6, n.11, p.383-388, 1916.

WIENDL, F.M. Efeitos da Radiação Gama sobre Zabrotes subfasciatus (Boh) Revista de Agricultura, Piracicaba, v.33, n.2, p.68, 1968.

WIENDL, F.M. Alguns usos e efeitos das radiaçōes gama em Zabrotes subfasciatus (Boh. 1833) (Col., Bruchidae). Piracicaba, 1969. 205p. Tese (Doutorado) - Escola Superior de Agricultura "Luiz de Queiroz", Universidade de São Paulo.

WIENDL, F.M. Efeitos da radiação gama em Sitophilus zeamais Mots., 1855. (Col., Curculionidae). Piracicaba, 1972. 156p. Tese (Livre Docência) Escola Superior de Agricultura "Luiz de Queiroz", Universidade de São Paulo.

Enviado para publicação em 20.08.1993

Aceito para publicação em 18.11.1993 